\title{
CFD simulation of dilute gas-solid two-phase flows with different solid size distributions in a curved $90^{\circ}$ duct bend
}

\author{
B. T. Kuan*
}

(Received 11 October 2004, revised 17 May 2005)

\begin{abstract}
CFD predictions of dilute gas-solid flow through a curved $90^{\circ}$ duct bend are performed. Flows with two different size distributions of glass spheres having mean diameters $66 \mu \mathrm{m}$ and $77 \mu \mathrm{m}$ are considered. The curved bend is square-sectioned $(150 \mathrm{~mm} \times 150 \mathrm{~mm})$ and has a turning radius of 1.5 times the duct's hydraulic diameter. Turbulent flow quantities at $\operatorname{Re}=15,000$ are calculated based on a Differential Reynolds Stress Model, while a Lagrangian particle tracking model predicts solids velocities. The model makes use of a modified shear-slip lift force formula which is consistent with experimental observation for $0.18<\operatorname{Re}_{p}<8$. The predictions are compared against experimental measurements taken using laser-Doppler Anemometry.
\end{abstract}

${ }^{*}$ Cooperative Research Centre for Clean Power from Lignite, Division of Minerals, CSIRO, Clayton, Australia. mailto: benny.kuan@csiro.au

See http://anziamj.austms.org.au/V46/CTAC2004/Kuan for this article, (c) Austral. Mathematical Soc. 2005. Published August 4, 2005. ISSN 1446-8735 
The study indicates that the predicted gas-solid flow behaviour near the outer wall is strongly dependent upon particle size fractions. Prediction quality deteriorates near the inner wall of the bend where local solids concentration diminishes and this points to a major limitation in the Lagrangian particle tracking methodology. The measured particle velocities at the inner wall region is found to be insensitive to the particle size distribution.

\section{Contents}

1 Introduction

C746

2 Test facility and flow conditions

C747

3 Theoretical background

C748

3.1 Gas phase . . . . . . . . . . . . . . . . . C748

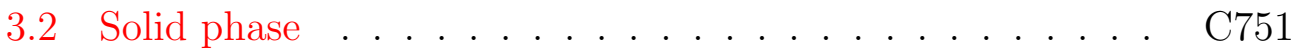

3.2.1 Shear-slip lift force . . . . . . . . . . . . . C752

3.2.2 Particle-wall interaction . . . . . . . . . . C C753

4 Results and discussions $\quad \mathbf{C 7 5 3}$

4.1 Gas phase . . . . . . . . . . . . . . C C753

4.2 Solid phase . . . . . . . . . . . . . . . C757

5 Conclusions $\quad \mathbf{C 7 6 0}$

References

C761 


\section{Introduction}

Elbows and bends are commonly used in pneumatic conveying systems to change flow direction so as to transport suspended material to the desired delivery point within a limited space. In the case of coal-fired power plants that operate on a continuous supply of pulverised coal to furnaces, maldistribution of pulverised fuel often occurs as coal particles are pneumatically transported from the mill through ducts consisting of numerous bends and straight sections.

In a coal-fired power plant utilising lignite, the mill-duct system is typically constructed from ducting components of large diameter-to-length ratios. Particle rope, which is a stratified layer of particles arising from severe nonuniformities in particle distribution, formed within the ductwork usually does not have sufficient straight duct length downstream to fully disperse and form a homogeneous fuel-air mixture prior to the fuel burners. This invariably makes it difficult for the plant operators to accurately control the amount of pulverised fuel supply to individual burners and hence maintain an optimal combustion condition inside the furnace. Furthermore, the non-uniform distribution of fuel across ducts severely complicates the measurement of fuel flow rate. Various sensing techniques have been developed by researchers (for example, Millen et al. [1], Ma and Yan [2]) to facilitate on-line measurement of solids mass flow rate in mill-ducts; however, so far none of them have been proven to work adequately for mill-duct flows subject to substantial particle roping.

Various experimental studies have explored particle roping in dust conveying systems with solids mass loading $>0.3$ (for example, Huber and Sommerfeld [3], Yilmaz and Levy [4]). It is generally accepted that the particle rope structure in such flow systems is very complex and is strongly affected by particle size, wall roughness, conveying velocity, and the radius of the bend. However, it is not clear from these studies whether the same also applies to a more dilute gas-solid flow regime (that is, solids mass loading $\ll 0.3$ ) that is 
common in the power plant mill ducts (for example, Founti and Klipfel [5]).

The primary objective of the present investigation is to test a CFD model of particle-laden flow for its ability to predict single and two-phase mill-duct flows in a $90^{\circ}$ bend. In the simulation, gas turbulence is solved with a differential Reynolds stress model (DRSM) whereas particle tracks are predicted through a Lagrangian approach taking into account one-way coupling, turbulence dispersion, and pressure gradient effects. The calculations also consider effects such as transverse lift force which encourages particle settling in the gas flow (Uijttewaal and Oliemans [6]), and surface roughness which may critically affect near-wall development of fine particle concentration (Sommerfeld et al. [7]).

Model validation makes use of laser-Doppler Anemometry (LDA) data that are directly obtained from tests conducted in CRC-Clean Power from Lignite's Advanced Laser Diagnostic Laboratory at CsIRO-Minerals. Flow measurements, including mean and fluctuating gas and solid velocities are performed on the duct's plane of symmetry.

\section{Test facility and flow conditions}

A schematic diagram of the test facility which is an open-circuit suction wind tunnel is shown in Figure 1. The square-sectioned $(150 \mathrm{~mm} \times 150 \mathrm{~mm})$ test section is constructed using $10 \mathrm{~mm}$ thick Perspex sheets, and the main components of the test facility include a $3.5 \mathrm{~m}$ horizontal straight duct, a $90^{\circ}$ bend with a turning radius of $225 \mathrm{~mm}$ and a $1.8 \mathrm{~m}$ vertical straight duct. All measurements are performed at numerous stations on the duct's plane of symmetry. The bulk air velocity $U_{b}=10 \mathrm{~m} / \mathrm{s}$.

Glass spheres with an average diameter of $66 \mu \mathrm{m}$ are released into the gas flow field from a fluidised-bed feeder. A digital balance underneath the fluidized-bed feeder monitors the rate at which the particles are consumed. 


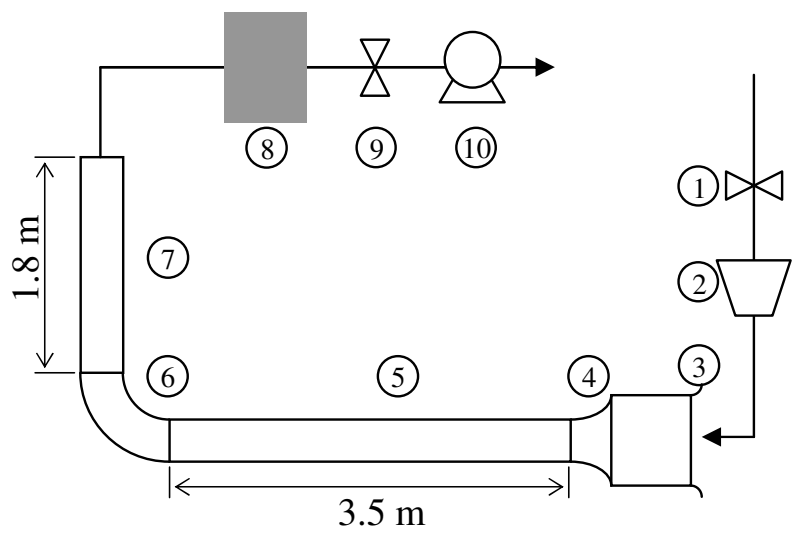

\begin{tabular}{|ll|}
\hline 1 & Feeder valve \\
2 & Fluidised bed feeder \\
3 & Bell mouth entry \\
4 & Converging duct \\
5 & Horizontal duct \\
6 & $90^{\circ}$ duct bend \\
7 & Vertical duct \\
8 & Baghouse dust collector \\
9 & Fan controller \\
10 & Centrifugal fan \\
\hline
\end{tabular}

FiguRE 1: Schematic diagram of the test facility.

This keeps the solid/gas mass loading ratio well below 0.01 thus ensuring a dilute gas-solid flow regime inside the test section. The same test is repeated with $77 \mu \mathrm{m}$ glass spheres. Particle size variations are determined through a Malvern particle size analysis and the result is shown in Figure 2. Also shown in the figure is the particle's Stokes number which is defined as a ratio of particle relaxation time to a turbulent time scale $k / \epsilon$ which is evaluated at the centre of the duct bend.

\section{Theoretical background}

\subsection{Gas phase}

Steady-state, isothermal gas flow properties and turbulence quantities are calculated numerically by solving a set of Reynolds averaged Navier-Stokes partial differential equations using a commercial CFD software CFX-4.4. The following differential Reynolds stress model (DRSM) as reported in Launder 


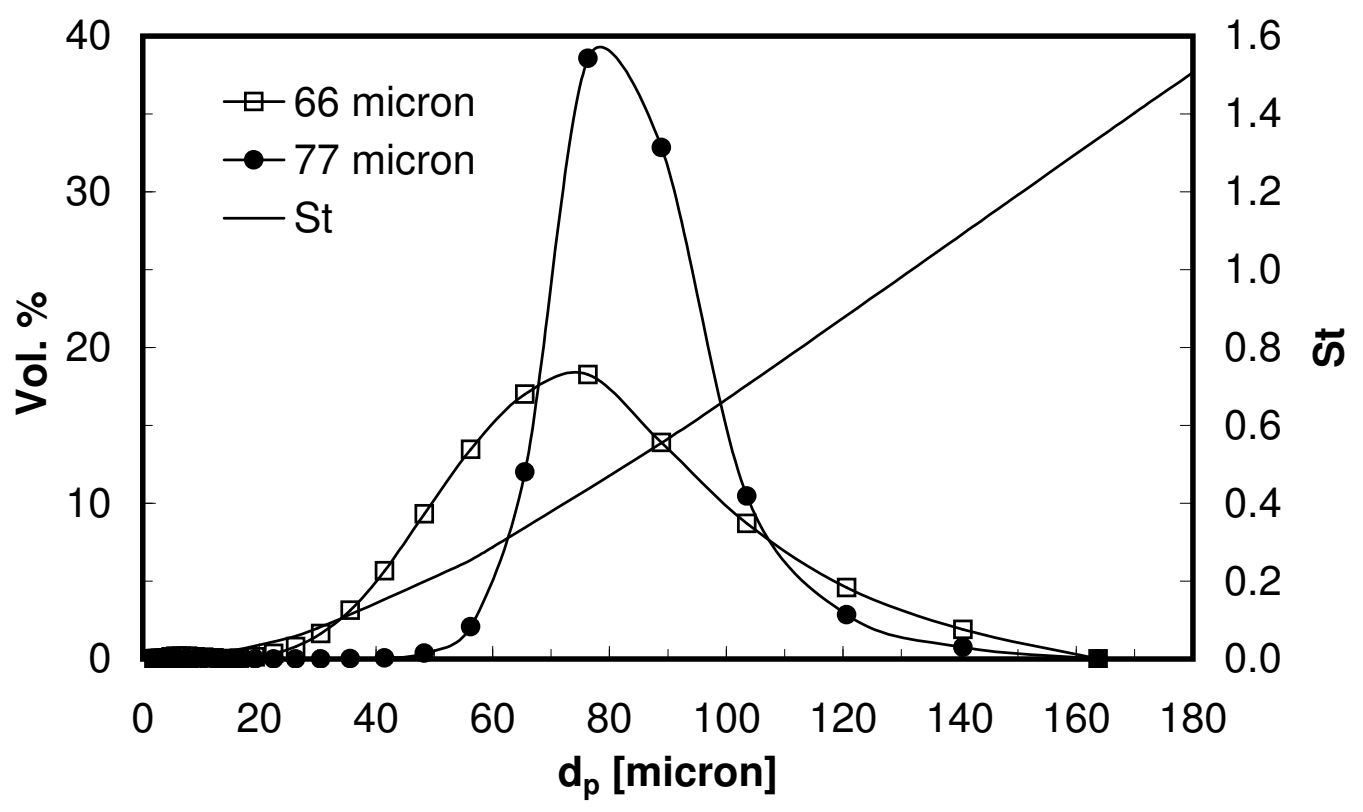

Figure 2: Measured particle size distributions.

et al. [8] was utilised:

$$
\begin{aligned}
& \frac{\partial \rho U_{k} \overline{u_{i}^{\prime} u_{j}^{\prime}}}{\partial x_{k}}=\frac{\partial}{\partial x_{k}}\left(C_{k} \rho \overline{u_{k}^{\prime} u_{l}^{\prime}} \frac{k}{\epsilon} \frac{\partial \overline{u_{i}^{\prime} u_{j}^{\prime}}}{\partial x_{l}}\right)+G_{i j}-\frac{2}{3} \rho \epsilon \delta_{i j}+\phi_{i j}, \\
& \frac{\partial \rho U_{k} \epsilon}{\partial x_{k}}=\frac{\partial}{\partial x_{k}}\left(C_{\epsilon} \rho \overline{u_{k}^{\prime} u_{l}^{\prime}} \frac{k}{\epsilon} \frac{\partial \epsilon}{\partial x_{l}}\right)+C_{\epsilon 1} \frac{\epsilon}{k} G_{k k}-C_{\epsilon 2} \frac{\rho \epsilon^{2}}{k}
\end{aligned}
$$

where $U$ and $u^{\prime}$ respectively represent mean and fluctuating velocities; and $\overline{u_{i}^{\prime} u_{j}^{\prime}}$ denotes the Reynolds stress tensor. The auxiliary functions are:

$$
\begin{aligned}
G_{i j} & =-\rho \overline{u_{i}^{\prime} u_{k}^{\prime}} \frac{\partial U_{j}}{\partial x_{k}}-\rho \overline{u_{j}^{\prime} u_{k}^{\prime}} \frac{\partial U_{i}}{\partial x_{k}} ; \\
\phi_{i j 1} & =-C_{1} \rho(\epsilon / k)\left[\overline{u_{i}^{\prime} u_{j}^{\prime}}-\overline{u_{k}^{\prime} u_{k}^{\prime}}\left(\delta_{i j} / 3\right)\right] ; \\
\phi_{i j 2} & =-C_{2}\left[G_{i j}-G_{k k}\left(\delta_{i j} / 3\right)\right] ;
\end{aligned}
$$




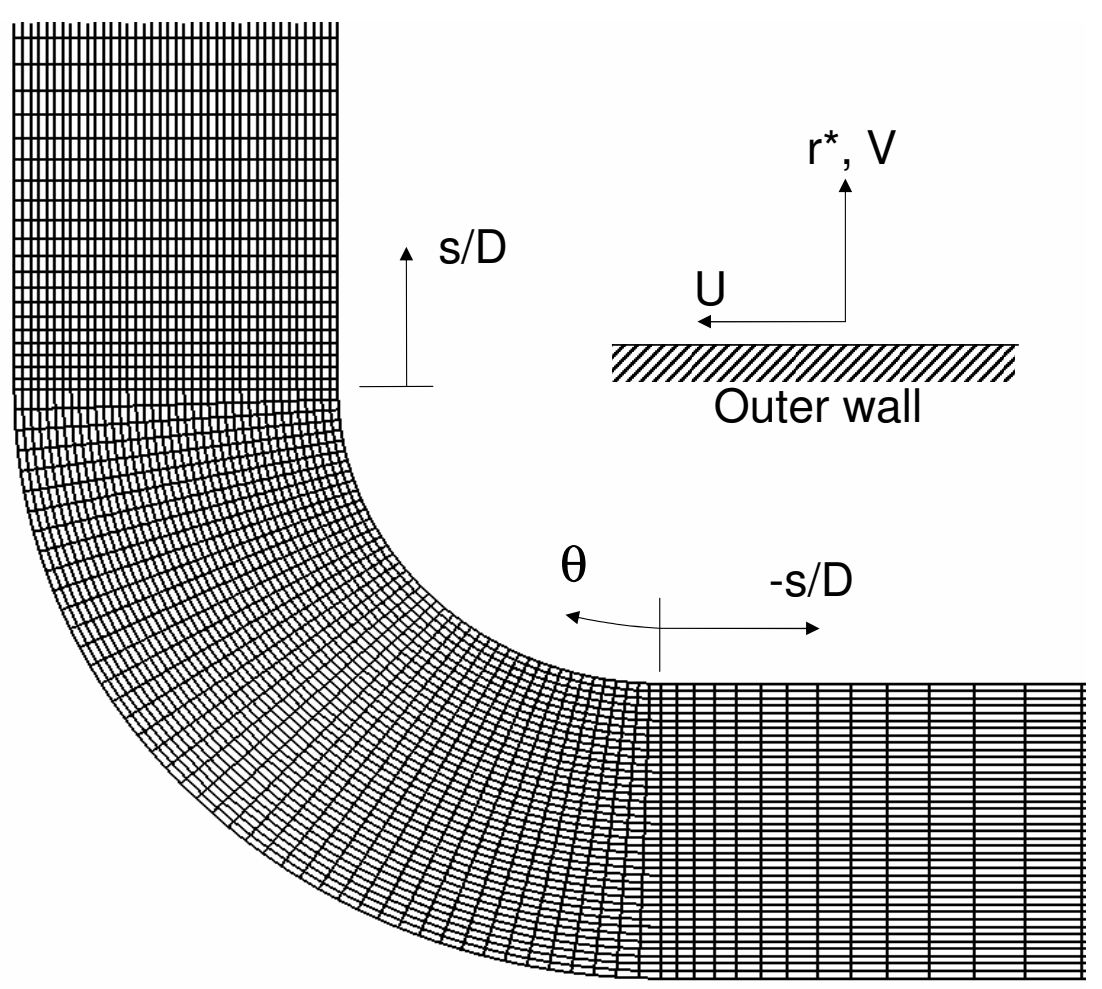

FiguRE 3: Body-fitted grid structure $(40 \times 40 \times 120)$ and the corresponding flow coordinate system.

$$
\begin{aligned}
\phi_{i j 3}= & {\left[C_{1}^{\prime} \frac{\epsilon}{k}\left(\overline{u_{k}^{\prime} u_{m}^{\prime}} \mathbf{n}_{\mathbf{k}} \mathbf{n}_{\mathbf{m}} \delta_{i j}-\frac{3}{2} \overline{u_{k}^{\prime} u_{i}^{\prime}} \mathbf{n}_{\mathbf{k}} \mathbf{n}_{\mathbf{j}}-\frac{3}{2} \overline{u_{k}^{\prime} u_{j}^{\prime}} \mathbf{n}_{\mathbf{k}} \mathbf{n}_{\mathbf{i}}\right)\right.} \\
& +C_{2}^{\prime}\left(\phi_{k m 2} \mathbf{n}_{\mathbf{k}} \mathbf{n}_{\mathbf{m}} \delta_{i j}-\frac{3}{2} \phi_{i k 2} \mathbf{n}_{\mathbf{k}} \mathbf{n}_{\mathbf{j}}-\frac{3}{2} \phi_{k j 2} \mathbf{n}_{\mathbf{k}} \mathbf{n}_{\mathbf{i}}\right) \\
& \left.+C_{3}^{\prime}\left(\phi_{k m 3} \mathbf{n}_{\mathbf{k}} \mathbf{n}_{\mathbf{m}} \delta_{i j}-\frac{3}{2} \phi_{i k 3} \mathbf{n}_{\mathbf{k}} \mathbf{n}_{\mathbf{i}}\right)\right] \frac{k^{1.5}}{C_{l} \epsilon x_{n}}
\end{aligned}
$$

where $\mathbf{n}_{\mathbf{k}}$ is a unit vector normal to the wall and $x_{n}$ is distance from the wall. $C_{k}, C_{\epsilon}, C_{1}, C_{2}, C_{1}^{\prime}, C_{2}^{\prime}, C_{3}^{\prime}, C_{l}, C_{\epsilon 1}$ and $C_{\epsilon 2}$ are model constants. 
The governing equations are discretised using a finite volume approach. The advection terms are approximated with a HYBRID differencing scheme [9] and the underlying pressure field is calculated using the well known SIMPLEC algorithm. The present HYBRID scheme is first-order accurate and hence diffusive in the presence of flow-to-grid skewness. A body-fitted grid (Figure 3) has thus been applied to minimise the skewness between the streamlines and the coordinate lines. A similar approach had been adopted in [10] and [11], and was found to produce accurate solutions comparable to that based on a higher-order difference scheme in complex flows.

When particles are introduced into a turbulent flow, they can either enhance or reduce the gas turbulence and thus affect the mean gas flow behaviour. However, in a dilute two-phase flow where particle volume fraction is in the order of $10^{-5}$ (that is, mass loading $\ll 0.02$ ), it is generally accepted that the transfer of particle momentum to the carrier-phase is negligible. Thus, particle and gas motion could be evaluated without consideration of particle-turbulence interaction (that is, one-way coupling). All two-phase flow calculations in this study use one-way coupling approach.

\section{$3.2 \quad$ Solid phase}

Instantaneous positions and velocities of the dispersed phase are solved from a set of ordinary differential equations in the Lagrangian domain:

$$
m_{p} \frac{d \mathbf{u}_{p}}{d t}=\mathbf{F}_{D}+\mathbf{F}_{g}+\mathbf{F}_{\mathrm{pg}}+\mathbf{F}_{A}+\mathbf{F}_{\mathrm{sl}}
$$

where subscript $p$ represents particle properties and subscripts $D, g, \mathrm{pg}$, $A$ and sl respectively denote force components arising from drag, gravity, flow pressure gradient, added mass effect and slip-shear lift. A detailed description of mathematical models for some of the force components considered in equation (7) is available from Fan and Zhu [12], and Huber and Sommerfeld [13]. 


\subsubsection{Shear-slip lift force}

Particles moving through a shear layer experience a transverse lift force due to a non-uniform pressure distribution as a result of unbalanced slip velocities on opposite sides of the particle. Saffman [14] derived an analytical expression for the shear lift force on a sphere for $\operatorname{Re}_{p} \ll 1$. Based on various studies by other researchers, Mei [15] later reformulated the Saffman's expression and extended it to higher $\mathrm{Re}_{p}$ regimes. To the best of the author's knowledge, Mei's model was never validated against any measured data due to a lack of reliable experimental studies at the time.

In the present study, Huber and Sommerfeld [13]'s shear-slip lift model, which stems from Mei's work, is modified to reflect the experimental findings of Mollinger et al. $[16,17]$ who directly measured the mean and fluctuating shear-slip lift force on a small sphere in the $0.18<\operatorname{Re}_{p}<8$ range. The resulting model is

$$
\begin{aligned}
& \mathbf{F}_{\mathrm{sl}}=\frac{\pi}{8} d_{p}^{3} \rho_{f} C_{\mathrm{sl}}\left(\left(\mathbf{u}_{f}-\mathbf{u}_{p}\right) \times \omega_{f}\right), \\
& C_{\mathrm{sl}}=C_{m} \frac{4.1126}{\sqrt{\operatorname{Re}_{s}}} f\left(\operatorname{Re}_{p}, \operatorname{Re}_{s}\right), \\
& f\left(\operatorname{Re}_{p}, \operatorname{Re}_{s}\right)= \begin{cases}(1-0.3314 \sqrt{\beta}) e^{-0.1 \operatorname{Re}_{p}}+0.3314 \sqrt{\beta}, & \operatorname{Re}_{p} \leq 40, \\
0.0524\left(\beta \mathrm{Re}_{s}\right)^{1 / 2}, & \operatorname{Re}_{p}>40\end{cases} \\
& \beta=0.5 \frac{\operatorname{Re}_{s}}{\operatorname{Re}_{p}}, \\
& \operatorname{Re}_{s}=\frac{\rho_{f} d_{p}^{2}\left|\omega_{f}\right|}{\mu}, \\
& \omega_{f}=\nabla \times \mathbf{u}_{f},
\end{aligned}
$$

where the modification factor is

$$
C_{m}=\frac{11}{\operatorname{Re}_{p}^{1 / 4}}
$$




\subsubsection{Particle-wall interaction}

The presently adopted particle-wall collisions model takes into account the contribution due to irregular particle-wall collisions. The model is primarily based on Matsumoto and Saito [18]'s study which categorises particle-wall collisions into either sliding or non-sliding collisions. The model also allows friction and restitution coefficients to vary as functions of wall material, particle size, and incident angle, which was observed in experiments, such as Schade et al. [19].

\section{Results and discussions}

\subsection{Gas phase}

Mean velocities for both gas and the dispersed phases are solved with DRSM on three grid systems having different cross-sectional cell densities (that is, $40 \times 40,60 \times 60$ and $80 \times 80$ cells). The results are compared in Figure 4 . Owing to space constraints, the graphs contain only selective profiles within and downstream of the $90^{\circ}$ bend.

According to the figure, the predicted gas flow is essentially grid independent, except at the inner duct wall just downstream of the bend exit. This corresponds to a region where local turbulence intensity grows rapidly as the bulk gas flow reattaches to the inner wall after the bend. However, such discrepancies are not unlikely to strongly influence the subsequent dilute particle track solution as will be discussed in the next section.

Based on the $80 \times 80$ grid, two additional calculations are performed utilising either a fully-turbulent upstream condition or flow data measured at $7 D$ prior to the elbow inlet. These are necessary because according to the experimental findings of Enayet et al. [22], strength and characteristics of secondary 


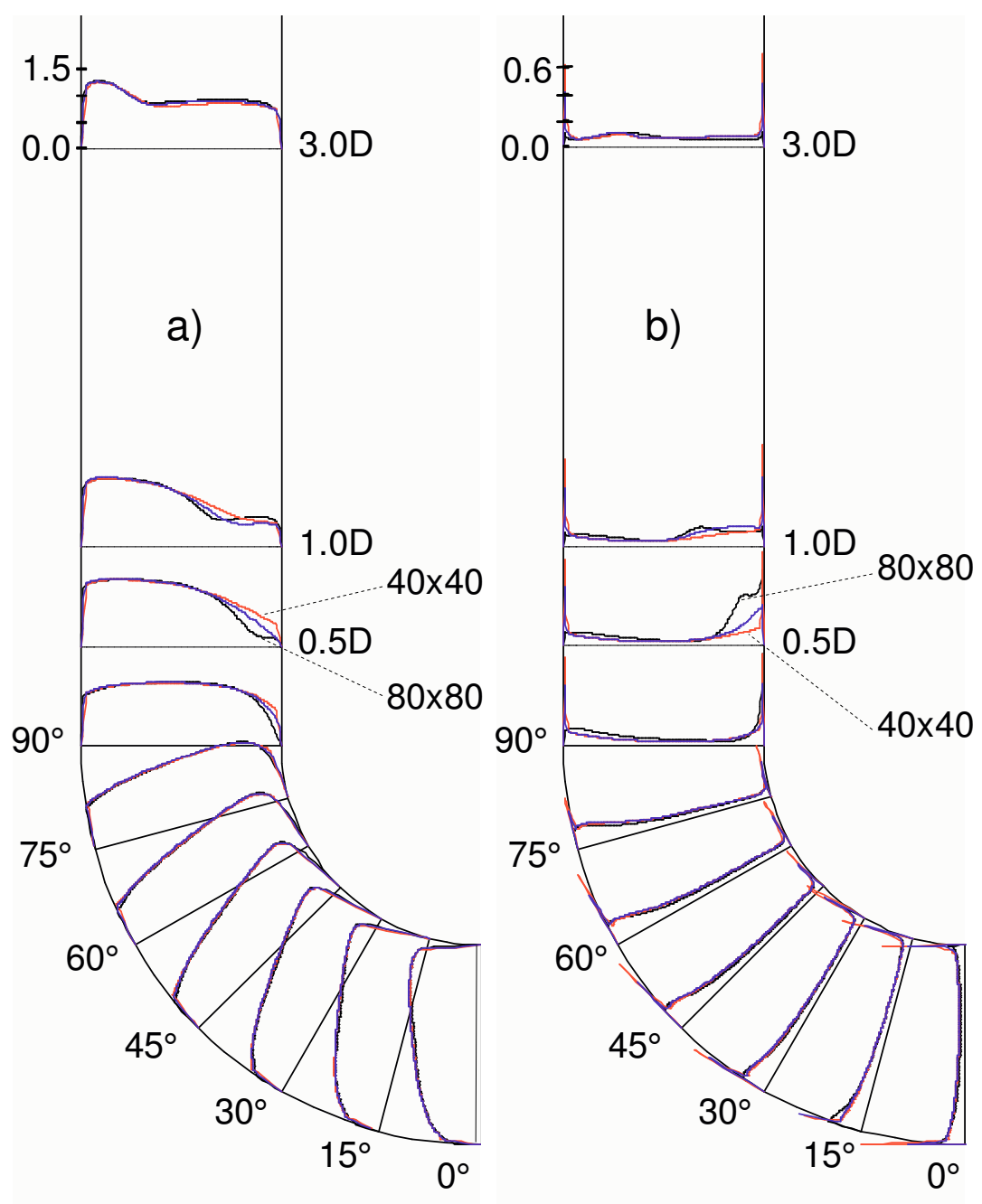

FIGURE 4: Sensitivity of the computed solution to grid density: a) normalised mean axial velocity $U / U_{b}$; b) turbulence intensity $\left|u^{\prime}\right| / \sqrt{U^{2}+V^{2}}$ : red, $40 \times 40$ grid; blue, $60 \times 60$ grid; black, $80 \times 80$ grid; $\bullet$, data. 

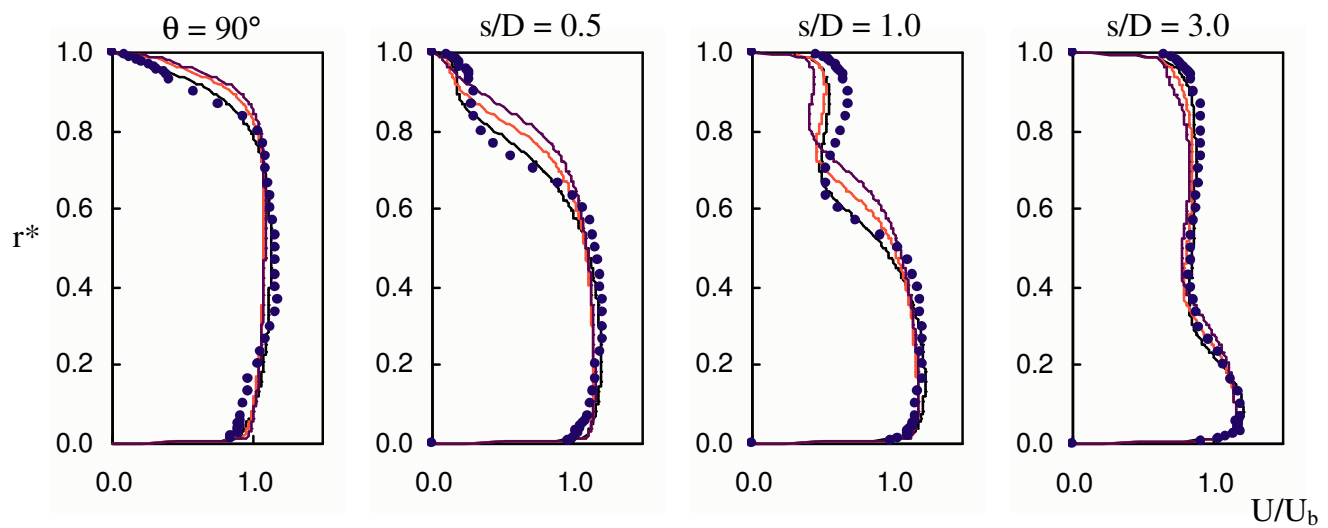

FIgURE 5: Sensitivity of the computed mean axial velocities $U / U_{b}$ to inlet conditions: black, DRSM + fully-developed inlet; red, $k-\epsilon$ model + fullydeveloped inlet; purple, $k-\epsilon$ model + measured inlet; $\bullet$, data.

flow motion following a duct bend are sensitive to inlet conditions. Further, our centre-plane flow measurement in the upstream duct indicates a gradual development of the core flow structure towards a fully-turbulent state. Owing to a difficulty in starting a DRSM simulation with only centre-plane profiles as initial conditions, the standard $\mathrm{k}-\epsilon$ turbulence model is applied. The numerical solution displays a dependence on inlet conditions though this seems to confine to a small area immediately downstream of the bend. As show in Figure 5, the predicted gas mean axial velocity profiles only differ appreciably at $s / D=0.5$ and 1.0. Further, the DRSM with a fully-developed inlet condition leads to a more satisfactory prediction of the mean gas flow.

The present flow problem is thus solved using DRSM on a $80 \times 80$ grid with a fully-developed initial condition.

As a means of ensuring numerical accuracy, the gas-phase flow field is also solved using one of the two higher-order differencing schemes: Van Leer MUSCL flux-limiter scheme [20]; and QUICK scheme [21]. As seen in Figure 6, 
the HYBRID solution is virtually identical to that given by the MUSCL and QUICK schemes, except at $0.5 D$ and $1.0 D$ downstream from the bend. In this region, the measured data seems to suggest the formation of a very small recirculation zone next to the inner wall. This is correctly reflected in the HYBRID solution. By contrast, both MUSCL and QUICK schemes over-predict the size of this recirculation zone.
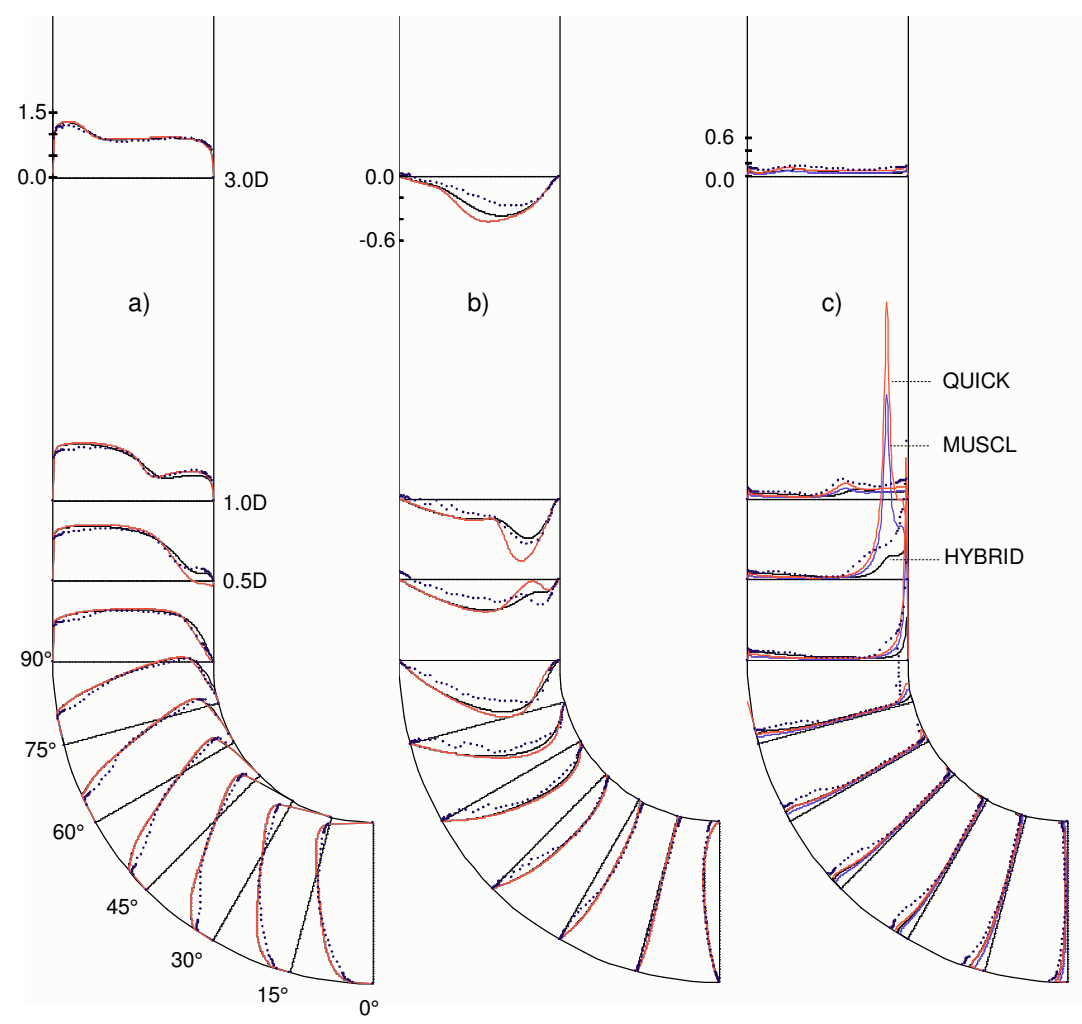

Figure 6: Comparison of predicted and measured flow properties a) normalised mean axial velocity $U / U_{b}$; b) normalised mean transverse velocity $V / U_{b}$; c) turbulence intensity $\left|u^{\prime}\right| / \sqrt{U^{2}+V^{2}}$ : red, QUICK; blue, MUSCL; black, HYBRID), $\bullet$, data. 
The predicted turbulence intensities are presented in Figure 6c. Again, the HYBRID solution provides a better qualitative representation of the turbulence structure. As compared to the laboratory data, it is found that within the bend, the gas flow near the outer wall decelerates in the presence of an adverse pressure gradient while the inner-wall flow accelerates under the influence of a favourable pressure gradient. Although this is a widely observed phenomenon in the published experiments, the DRSM is unable to predict this flow behaviour accurately. At the outer wall, the model grossly over-predicts the mean gas axial velocity whereas the mean axial velocity near the inner wall is under-predicted by a maximum of $11 \%$. However, the numerical model is able to capture the transport of the core flow normal to the duct wall correctly. The turbulence intensities have all been normalised by the planar mean gas velocities, that is, $\sqrt{U^{2}+V^{2}}$.

The application of DRSM leads to a good qualitative representation of the underlying turbulence structure. Quantitatively, the predicted turbulence fluctuation could only reach $60 \%$ of the measured value at the wall.

\subsection{Solid phase}

Lagrangian particle tracking with one-way coupling is applied to evaluate particle dynamics within the elbow duct because solid motion has negligible influence on the carrier fluid flow. Based on the DRSM result, a total of 100,000 particle tracks are calculated for the given particle size distributions. The results are presented in Figure 7.

In general, finer particles tend to better follow the underlying gas motion. But in the second half of the bend, even the $66 \mu \mathrm{m}$ particles experience a nonnegligible gas-solid segregation as a result of the centrifugal effect. This is characterised by a local drop in particle concentration near the inner wall and is well reflected in the prediction. The calculation suggests a very minor slip between the two phases over a large part of the duct centre plane whereas 

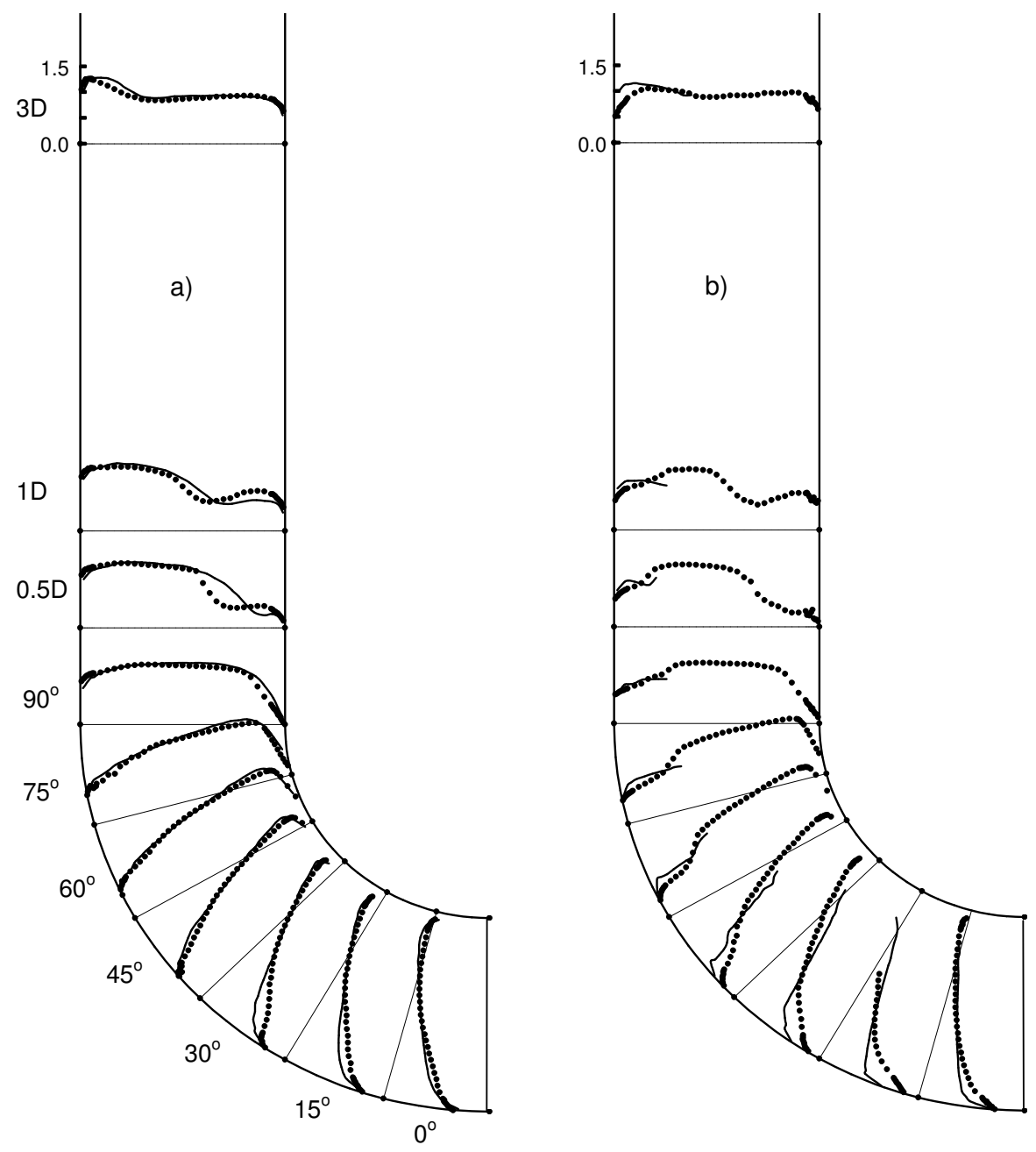

FiguRE 7: Normalised mean particle velocity profiles: a) axial velocity $U_{p} / U_{b}$ for $66 \mu \mathrm{m}$ particles; b) axial velocity $U_{p} / U_{b}$ for $77 \mu \mathrm{m}$ particles: - , prediction; •. data. 
noticeable gas-solid separation is observed immediately downstream of the bend at $0.5 D$ (Figure $7 \mathrm{a}$ ).

According to the measured data, particles lead the gas near the outer wall at $45^{\circ}, 60^{\circ}, 75^{\circ}$ and $90^{\circ}$ into the bend. The 'negative' slip velocities are partially caused by the presence of coarse particles at these measurement locations. In addition to the 'no-slip' condition that exists between the gas phase and the wall, the coarser particles are slower to respond to changes in the local gas flow field; thus the measured particle velocity near the outer wall could well exceed the local gas velocity. This feature is not reflected in the calculated profiles (that is, no gas-solid separation) because the predicted adverse pressure gradient at the outer wall is too weak to decelerate the gas adequately (see Figure 6a).

For particles having a mean diameter of $77 \mu \mathrm{m}$, the present particle tracking model tends to over-predict particle axial velocities at the outer wall in the first half of the bend (Figure 7b). This may point to a deficiency in the particle-wall interaction model. However, outside this region good agreement between the measured and predicted particle velocity is achieved.

Near the inner walls, concentration of the predicted particle tracks become so low that the averaged velocity profiles discontinue in the locality. This phenomenon persists until $5 D$ downstream from the bend. Such a decline in particle population reduces the effective sample space within which statistical averaging of local particle velocities are carried out. Thus, the 'averaged' particle velocity profiles away from the outer wall are no longer statisticallymeaningful. This problem is not as severe for the $66 \mu \mathrm{m}$ case because the particle tracks calculated in the preceding section contain a greater fraction of fine particles (see Figure 2). This leads to a larger number of predicted particle tracks in the inner-wall region and hence a better resolution.

In an attempt to raise the presence of fine particles in the numerical calculation and hence improve the resolution of the predicted particle tracks in the inner wall region for the $77 \mu \mathrm{m}$ case, one million particles have been 
tracked but this did not result in any noticeable improvement. A much larger number of particles thus need to be tracked in order to produce an acceptable particle data resolution.

\section{Conclusions}

Two-phase LDA measurements as well as numerical simulations are performed for a curved $90^{\circ}$ duct bend. The experimental data has been applied to evaluate the result of a CFD simulation in an effort to identify areas where further work is necessary to improve the accuracy of numerical prediction for flows with dilute solid suspension. We find the following.

1. Gas turbulence solution based on DRSM is unable to capture the correct pressure gradient effects that prevail within the bend; the predicted turbulence intensity only bears qualitative resemblance to the measured distribution.

2. In cases where fine particles make up a substantial portion of the solid phase, slip between the gas and the solids tend to become negligible. Hence quality of the gas-phase solution is crucial to an accurate prediction of fine particle tracks.

3. The coarse particle track solutions are sensitive to particle-wall interaction models as well as particle size fractions.

Acknowledgment: I gratefully acknowledges the financial and other support received for this research from the CRC-Clean Power from Lignite, which is established and supported under the Australian Government's Cooperative Research Centre program. I also appreciate William Yang's effort in post-processing the laboratory data and Hasan Khan for proofreading the manuscript. 


\section{References}

[1] M. J. Millen, B. D. Sowerby, P. J. Coghill, J. R. Tickner, R. Kingsley and C. Grima. Plant tests of an on-line multiple-pipe pulverized coal mass flow measuring system. Flow Measurement and Instrumentation, 11, 153-158, 2000.

http://dx.doi.org/10.1016/S0955-5986(00)00013-3 C746

[2] J. Ma, and Y. Yan. Design and evaluation of electrostatic sensors for the measurement of velocity of pneumatically conveyed solids. Flow Measurement and Instrumentation, 11, 195-204, 2000. http://dx.doi.org/10.1016/S0955-5986(00)00019-4 C746

[3] N. Huber and M. Sommerfeld. Characterization of the Cross-Sectional Particle Concentration Distribution in Pneumatic Conveying Systems. Powder Technology, 79, 191-210, 1994.

http://dx.doi.org/10.1016/0032-5910(94)02823-0 C746

[4] A. Yilmaz and E. K. Levy. Roping phenomena in pulverized coal conveying lines. Powder Technology, 95, 43-48, 1998.

http://dx.doi.org/10.1016/S0032-5910(97)03314-7 C746

[5] M. A. Founti and A. S. Klipfel. Numerical simulation of pneumatic transport and erosion wear in the distribution ducts in large lignite power plants. ASME Fluids Engineering Conference, FED-Vol. 236, 717-724, 1996. C747

[6] W. S. J. Uijttewall and R. V. A. Oliemans. Particle dispersion and deposition in direct numerical and large eddy simulations of vertical pipe flows. Physics of Fluids, 8(10), 2590-2604, 1996.

http://dx.doi.org/10.1063/1.869046 C747

[7] M. Sommerfeld, N. Huber, and P. Wächter. Gas-Solid Flows. in D. E. Stock, M. W. Reeks, Y. Tsuji, M. Gautam, E. E. Michaelides, and 
J. T. Jurewicz, editors, ASME Fluids Engineering Conference, FED-Vol. 166, 183-191, 1993. C747

[8] B. E. Launder, G. J. Reece, and W. Rodi. Progress in the development of a Reynolds stress turbulence closure. Journal of Fluid Mechanics, 68(3), 537-566, 1975. C749

[9] D. G. Lilley and D. L. Rhode. A computer code for swirling turbulent axisymmetric recirculating flows in practical isothermal combustor geometries. NASA Contractor Report CR3442, 1982. C751

[10] W. Shyy and M. Correa. A Systematic comparison of several numerical schemes for complex flow calculations. AIAA 23rd Aerospace Sciences Meeting, January 14-17, Nevada, AIAA-85-0440, 1985. C751

[11] S. Hogg and M. A. Leschziner. Computation of highly swirling confined flow with a Reynolds stress turbulent model. AIAA Journal, 27(1), 57-63, 1989.

http://pdf . aiaa.org/jaPreview/AIAAJ/1989/PVJAPRE10094.pdf C751

[12] L. S. Fan and C. Zhu. Principles of gas-solid flows. Cambridge University Press, Cambridge, UK, 1998. C751

[13] N. Huber and M. Sommerfeld. Modelling and numerical calculation of dilute-phase pneumatic conveying in pipe systems. Powder Technology, 99, 90-101, 1998.

http://dx.doi.org/10.1016/S0032-5910(98)00065-5 C751, C752

[14] P. G. Saffman. The lift on a small sphere in a slow shear flow. Journal of Fluid Mechanics, 22(2), 385-400,1965. C752

[15] R. Mei. An approximate expression for the shear lift force on a spherical particle at finite Reynolds number. International Journal of Multiphase Flow, 18(1), 145-147, 1992.

http://dx.doi.org/10.1016/0301-9322(92)90012-6 C752 
[16] A. M. Mollinger, F. F. M. Nieuwstadt, and J. M. Bessem. A new device to measure the lift force on a particle in the viscous sublayer. Measurement in Science and Technology, 6, 206-213, 1995. http://www.iop.org/EJ/abstract/0957-0233/6/2/013 C752

[17] A. M. Mollinger and F. T. M. Nieuwstadt. Measurement of the lift force on a particle fixed to the wall in the viscous sublayer of a fully developed turbulent boundary layer. Journal of Fluid Mechanics, 316, 285-306, 1996. C752

[18] S. Matsumoto and S. Saito. Monte Carlo simulation of horizontal pneumatic conveying based on the rough wall model. Journal of Chemical Engineering of Japan, 3(1), 223-230, 1970. C753

[19] K. P. Schade, H. J. Erdmann, Th. Hadrich, H. Schneider, Th. Frank, and K. Bernert. Experimental and numerical investigation of particle erosion caused by pulverised fuel in channels and pipework of coal-fired power plant. Powder Technology, 125, 242-250, 2002.

http://dx.doi.org/10.1016/S0032-5910(01)00512-5 C753

[20] B. Van Leer. Towards the ULTIMATE conservation difference scheme. II. Monotonicity and conservation combined in a second order scheme. Journal of Computational Physics, 14, 361-370, 1974. http://dx.doi.org/10.1016/0021-9991(74)90019-9 C755

[21] B. P. Leonard. A stable and accurate convective modelling procedure based on quadratic upstream interpolation. Computational Methods in Applied Mechanical Engineering, 18, 59-98, 1979. http://dx.doi.org/10.1016/0045-7825(79)90034-3 C755

[22] M. M. Enayet, M. M. Gibson, A. M. K. P. Taylor, and M. Yianneskis. Laser-Doppler measurements of laminar and turbulent flow in a pipe bend. International Journal of Heat and Fluid Flow, 3(4), 213-219, 1982. http://dx.doi.org/10.1016/0142-727X (82)90024-8 C753 